Table 1. Percentage Post-Eugragnce Damping-off of Sendlings OF FIVE VEGETABLES INOCULATED AT SOIL-LEVEL WITH AGAR DISKS CONTAINING $\boldsymbol{R}$. solani, $\boldsymbol{P}$. intermedium OR Fusarium SP. (MEDIUM SOIL MOISTURE-CONTENT; TEMPERATURE $0-30^{\circ}$ C.)

\begin{tabular}{|l|c|c|c|}
\hline \multicolumn{1}{|c|}{ Vegetable } & \multicolumn{2}{|c|}{ Percentage $\begin{array}{c}\text { post-emergence } \\
\text { induced by }\end{array}$} \\
\hline & R. solani & P. intermedium & Fusarium sp. \\
\hline Peas & 100 & 0 & 9 \\
Cucumber & 100 & 0 & 0 \\
Cabbage & 87 & 14 & 8 \\
Caulifiower & 100 & 20 & 12 \\
Vetch & 100 & 0 & 12 \\
\hline
\end{tabular}

from the fungus were kept. All seedlings were kept in the greenhouse where the temperature ranged between $0^{\circ}$ and $30^{\circ} \mathrm{C}$. The seedlings were observed for a period of four weeks and the number of dampedoff seedlings was recorded. The results of this experi. ment are set out in Table 1. Seedlings inoculated with disks of agar free from fungi remained healthy throughout the experimental period and hence their results are omitted from Table 1.

Table 1 shows that $P$. intermedium and Fusarium sp. are weak pathogens so far as the post-emergence phase of damping-off disease of the five test vegetables is concerned. On the other hand, $R$. solani proved to be a destructive damping-off causal organism.

From the results of this and the previous experiment it can be safely concluded that, contrary to the findings of Al-Adhami ${ }^{1}, R$. solani is the main causal organism of both pre- and post-emergence phases of damping-off disease of cabbage and cauliflowers as well as the other three vegetables tested. It may be worth mentioning in this respect that Pythium de baryanum, which was reported by AlAdhami $^{1}$ as the causal organism of damping-off of cabbage and cauliflower, was never met with on the isolation plates throughout this investigation.

M. K. TOLBA*

A. L. SALIM

Department of Botany,

College of Arts and Science, Baghdad, Iraq.

* Permanent address : Department of Botany, University of Cairo. ${ }^{2}$ Al-Adhami, A. R., Tech. Bull, No. 17, Ministry of Agriculture, Iraq (1953)

${ }^{2}$ Tolba, M. K., Proc. Egypt Acad. Sci., 8, 44 (1952).

\section{Development of Female Schistosoma spindale in the Guinea Pig}

Previous workers ${ }^{1}$ have reported that only the male of Schistosoma spindale develops in the guinea pig. Fairley, Mackie and Jesudasan, who carried out a detailed investigation, suggested that "some peculiar host reaction to the less robust. female schistosome probably underlies this phenomenon in the case of $S$. spindale". A comprehensive study was undertaken of this apparent immunity to only one sex of a metazoan parasite, as a result of which it has been found that female $S$. spindale also develops in the guinea pig.

Twenty-four guinea pigs of both sexes and one and a half months to two years of age were exposed to cercaria of $S$. spindale discharged by naturally infected Indoplanorbis exustus. The snails were collected during August, September and October 1954 from a local tank frequented by infected puffaloes. Each animal was exposed to the cercarix percutaneously by keeping three-fourths of its body immersed in water containing the larvæ. Two-litre glass jars were used for the purpose. The duration of exposure was about three hours. The number of cercariæ employed per guinea pig varied from 8,000 to 50,000 , and that of the snails furnishing the cercariæ from 22 to 77 .

One guinea pig died early and another could not be examined for other reasons. Of the remaining twentytwo animals, which either died or were autopsied 33-153 days after exposure, seventeen provided conclusive evidence of the development of female $S$. spindale and two furnished inconclusive evidence. The male $S$. spindale, however, developed in all the twenty-two animals. Among the seventeen conclusive cases of infection with female $S$. spindale, mature females and pseudotubercles containing the characteristic ova in liver, intestine or lung were obtained in eight, immature females in four and only pseudotubercles containing the ova in the remaining five. The two inconclusive cases contained the pseudotubercles, but the ova inside were degenerate and not very distinct. The maximum number of immature females recovered was 440 , while that of mature females was eleven. The live immature females were filamentous and the edges of the body tended to infold ventrally, forming a 'pseudogynæ. cophoric canal'. The ventral sucker was compara. tively more developed. The females were shorter than the males. The maximum number of uterine ova was three. The earliest period in which the females attained maturity, as evidenced by the appearance of ova in the uterus and host tissue, was 48 days; but in a few cases they had remained immature up to 99 days after exposure. Ova were never detected in the fæces of the infected animals.

The present study thus shows that the female of Schistosoma spindale may also develop in the guinea pig, which therefore does not possess total immunity to the female schistosome. But this species of animal is not a very suitable host of the female schistosome, as is evidenced by the facts that in a few cases the females did not develop at all; in others they remained immature up to more than three months and in still others they were apparently destroyed after the production of ova. This variability in the susceptibility of guinea pigs to the infection may be explained on the basis of individual immunity of varying degree. The difference between the present observations and those of the previous workers may mostly be attributable to the variability in the susceptibility of the host. It is, however, possible that the infection reported by Fairley et al. was not purely unisexual, for the immature filamentous worms recorded by them were, perhaps, only the immature females of $S$. spindale. As mentioned above, similar worms were found in the present investigation also.

A detailed account of this work will be published elsewhere. I am grateful to Mr. L. Sahai, director of this Institute, for his interest in the work, and to Dr. H. D. Srivastava, head of the Division of Parasitology, for providing the requisite facilities.

Division of Parasitology, S. C. DuTr

Indian Veterinary Research Institute, Izatnagar, India. Feb. 28.

${ }^{2}$ Liston, W. G., and Soparkar, M. B., Ind. J. Med. Res, 5, 567 (1918). Fairley, N. H., Mackie, E. P., and Jesudasan, F., Ind. Med. Res. Mem., Husb., 4, 1 (1934). 